\title{
Subconscious manipulation of pain expectation can modulate cortical nociceptive processing
}

Gwyn N Lewis*1 PhD, Adam Leys ${ }^{1}$ BHSc, David A Rice ${ }^{1,2} \mathrm{PhD}$, Peter J McNair ${ }^{1} \mathrm{PhD}$ ${ }^{1}$ Health and Rehabilitation Research Institute, AUT University, Auckland, New Zealand ${ }^{2}$ Waitemata Pain Service, Department of Anaesthesiology and Perioperative Medicine, Waitemata District Health Board, Auckland, New Zealand

* Author for correspondence Health and Rehabilitation Research Institute AUT University, Private Bag 92006

Auckland 1142

New Zealand

Ph: +6499219999

Fax: +64 99219620

email: gwyn.lewis@aut.ac.nz 


\section{Abstract}

Objective: To determine if manipulation of the expectation of pain inhibition can enhance the efficacy of conditioned pain modulation in healthy participants. Methods: A conditioned pain modulation paradigm was used to investigate the effect of psychological manipulation of expectation on pain inhibition. In 19 healthy males, the lower limb nociceptive flexion reflex was elicited in isolation (test stimulus) and during application of two forms of conditioning stimuli. Following application of the first conditioning stimulus (CS1), the participants were informed that the subsequent conditioning stimulus (CS2) would elicit a greater amount of inhibition of test pain compared to the first. Lower limb flexion reflex size, perceived pain ratings of the test stimulus, and ratings of expected pain modulation were obtained for both test and conditioning protocols. Results: The inhibition of perceived pain was significantly greater with CS2 compared to CS1; however, there was no significant difference in inhibition of nociceptive flexion reflex size or the participant's reported expectation of pain modulation between the two conditioning stimuli.

Discussion: As perceived pain inhibition was enhanced but flexion reflex size unchanged following the intervention, we suggest that the intervention gave rise to an inhibition of ascending nociceptive information at a supraspinal level resulting in reduced pain perception without influencing spinal level processing of nociceptive input. The finding that conditioned pain modulation can be enhanced is of relevance to clinical pain populations who commonly show impaired inhibition.

Key words: conditioned pain modulation, expectation, flexion reflex, psychology 


\section{Introduction}

Conditioned pain modulation paradigms are often used to assess the efficacy of our endogenous pain inhibitory system. In such paradigms, a painful test stimulus is applied on its own and during the application of a secondary, painful conditioning stimulus to a remote part of the body. The pain associated with the test stimulus is reduced during the application of the conditioning stimulus, with the extent of modulation a reflection of the efficacy of the inhibitory system. The response is assumed to be mediated, in part, by neurons associated with the diffuse noxious inhibitory control (DNIC) system, a neuronal group residing in the caudal medulla whose axons terminate on multireceptive dorsal horn neurons throughout the spinal cord..$^{1,2}$ The conditioned pain response also includes a supraspinal component that is postulated to involve cortico-cortico effects mediated by activity in the orbitofrontal cortex and amygdala.3,4

Current research indicates that the efficacy of conditioned pain modulation is influenced by a person's expectations regarding the change in pain. ${ }^{5-8}$ From an observational study, Larivière et al. ${ }^{7}$ provided an initial indication of a relationship between the reported expectation of inhibition and the actual conditioned pain modulation response in healthy individuals. Goffaux and colleagues ${ }^{6}$ performed an experimental trial that involved cognitive manipulation of the participants' expectation. In this study, one group of participants were told to expect analgesia with the conditioning stimulus and a second group were told to expect hyperalgesia. The analgesia expectancy group experienced a $77 \%$ decrease in perceived test pain during the conditioning stimulus, whereas the hyperalgesia expectancy group experienced no effect of conditioning. These results were reflected in recordings of cortical and spinal level nociceptive processing, indicating that 
perceived expectations of pain can modulate nociceptive input directly at the level of the spinal cord through modulation of descending inhibitory pathways.

Goffaux et al. ${ }^{6}$ did not include a control condition in which expectations were not manipulated. Thus, it is difficult to determine if the group expecting analgesia showed an enhanced inhibitory response over what would have occurred if the participants had not received the intervention. Demonstrating that the efficacy of conditioned pain modulation can be facilitated would be of obvious clinical benefit to chronic pain populations who routinely show an impaired modulatory response $\mathrm{e}^{9,10}$ and may enhance our understanding of how positive expectation of pain relief augments the analgesic response. Therefore, the aim of the current study was to determine if manipulation of expectations could increase the efficacy of conditioned pain modulation within the same group of participants. Pain modulation was evaluated firstly in a naïve condition without expectation manipulation and a second time following an intervention designed to enhance the expectation of pain inhibition. It was hypothesised that the second evaluation of conditioned pain modulation would result in greater expectation of pain relief and a greater actual inhibition of test stimulus pain.

\section{Materials and Methods}

\section{Study design}

A randomised, cross-over experimental design was implemented using two types of conditioning stimuli in a single group of participants. Two different conditioning stimuli were used to minimise the outcome of the first evaluation influencing the expectation of 
the second conditioning stimulus. Conditioned pain modulation was assessed once with each conditioning stimulus, with the order of conditioning stimuli randomised between participants. The randomised, cross-over design meant that expectations for analgesia were manipulated for the one type of conditioning stimulus for some participants and for the other type of conditioning stimulus for the remaining participants. The room temperature was maintained at $23^{\circ} \mathrm{C}$ throughout testing. Participants were asked to refrain from consuming any product containing caffeine, alcohol, or nicotine and were asked not to participate in any strenuous activities for four hours before testing.

\section{Participants}

Nineteen healthy males volunteered for the study (mean age 31 \pm 5 years, range $22-43$

years). Participants were excluded if they had any of the following: current pain or history of chronic pain, knowledge regarding the function of the DNIC system, any musculoskeletal conditions that restricted the ability to perform the test procedures, or a severe cardiorespiratory condition. Additionally, participants aged 50 and over were excluded due to the reduction in conditioned pain modulation efficiency with age $\mathrm{e}^{7,11}$ and females were excluded to remove potential bias of hormonal fluctuations at different phases of the menstrual cycle. ${ }^{12}$ Ethical approval was gained from the institutional ethics committee and written informed consent was received from all participants.

\section{Test stimulus}

Participants stood with their left leg on a footstool and their right leg hanging passively. To elicit the nociceptive flexion reflex, electrocutaneous stimulation was applied to the medial plantar nerve of the right leg using a bipolar electrode (Nicolet Biomedical) filled with 
conductive gel. The electrode was placed on the medial plantar aspect of the foot $2 \mathrm{~cm}$ proximal to the metatarsophalangeal joint and in line with the first metatarsal. Each stimulus consisted of a train of five stimuli (1 ms duration) at $250 \mathrm{~Hz}^{13}$ that were delivered by a constant current stimulator (DS7A, Digitimer, UK). Participants were initially exposed to 10 stimuli at varying intensities to familiarise them with the procedure and reduce potential anxiety. The nociceptive flexion reflex threshold was then found using a standardised staircase method. ${ }^{14}$ Stimulation intensity was increased from $0 \mathrm{~mA}$ in $4 \mathrm{~mA}$ increments until a reflex was observed and then decreased in $2 \mathrm{~mA}$ steps until a reflex was no longer evident. The intensity was further increased and decreased four more times in 1 $\mathrm{mA}$ increments until a reflex appeared and disappeared twice. The final four stimulation intensities were recorded and averaged to calculate reflex threshold. Ten test stimuli were then applied 8-12 seconds apart at $120 \%$ of reflex threshold. If a reflex was not elicited following each stimulus, the stimulus intensity was increased in $10 \%$ intervals until a response was consistently elicited and a further 10 test stimuli were delivered at this intensity. Perceived pain ratings associated with each of the ten test stimuli were obtained using a numerical pain rating (NPR) from $0-100$, where $0=$ no sensation, $1=$ just noticeable sensation, $25=$ uncomfortable, $50=$ painful, $75=$ very painful, $100=$ maximum tolerable pain .

\section{Nociceptive flexion reflex recording}

Surface electromyography (EMG) was used to record the reflex in a knee flexor muscle using disposable bipolar electrodes (Myotronics Norotrode). The recording electrode was placed $10 \mathrm{~cm}$ superior to the popliteal fossa over the biceps femoris of the right leg. A unipolar ground electrode (3M Red Dot) was placed over the midshaft of the right tibia. The EMG signals were collected using a data acquisition board (Micro 1401) and Signal 
software (CED, Cambridge, UK). Signals were amplified (x1000), filtered (10-1000 Hz), and sampled at $5000 \mathrm{~Hz}$. A reflex response was defined as present when the EMG response within the 85-150 ms window post-stimulus surpassed mean EMG activity $70 \mathrm{~ms}$ prestimulus ( -80 to $-10 \mathrm{~ms}$ ) window by 1.4 standard deviations..$^{15}$ Trials were removed and repeated if the background EMG did not show muscle quiescence.

\section{Conditioning stimuli}

The two conditioning stimuli used were a cold pressor test and an ischaemic arm test. The cold pressor test was performed by immersing the left hand into an ice water bath $\left(12 \pm 1^{\circ} \mathrm{C}\right)$ up to the wrist line. Pain was monitored every 15 seconds to establish if the stimulus had become painful (NPR $\geq 60$ ). If the NPR was $\geq 60$ after 1 minute, 10 test stimuli were given with the hand remaining submerged. If a NPR of $\geq 60$ had not been reached, participants continued with their hand in the ice water bath until a rating of $\geq 60$ was reported and then received the 10 test stimuli.

The ischaemic arm test was performed by elevating the left arm for one minute to induce venous blood drainage. During this minute, a $15 \mathrm{~cm}$ sphygmomanometer cuff was attached slightly proximal to the cubital fossa and inflated to $250 \mathrm{mmHg}$. The arm was then returned to a horizontal position and rested on a raised pillow on the participant's lap. Participants were instructed to perform 20 wrist extensions with a $2.5 \mathrm{~kg}$ weight with a $1 \mathrm{~s} \mathrm{hold} \mathrm{in} \mathrm{full}$ extension and a $1 \mathrm{~s}$ hold in full relaxation. After 20 extensions participants rated their pain using the NPR. If the rating was $\geq 60$ then participants stood up and 10 test stimuli were delivered. If a NPR $\geq 60$ had not been reached then participants were instructed to do 
further wrist extensions until a NPR $\geq 60$ was reported, following which participants ceased the exercise and had 10 test stimuli delivered.

\section{Experimental protocol}

Baseline nociceptive flexion reflexes and subjective pain ratings were obtained as outlined above (B1). The procedure for the first conditioning stimulus was then explained to the participants using standardised wording. It was indicated that the conditioning stimulus may either increase or decrease the pain in the foot from the test stimulus as the current research evidence for this was uncertain. After the explanation, participants were asked to rate their expectation of how the conditioning stimulus would influence the test pain using a rating scale from -100 to +100 , where $-100=$ completely inhibit pain, $0=$ no change in pain, and $100=$ strongly magnify pain.6,16 The first randomly assigned conditioning stimulus was then applied and the 10 test stimuli repeated (CS1).

A 20-minute break followed CS1 during which the participants were seated and the procedure for the second conditioning stimulus was explained using standardised wording. In this case, participants were informed that the conditioning stimulus should significantly reduce the intensity of pain felt from the test stimulus and that there were "numerous research trials" to provide evidence for this result. The explanation was delivered in such a way to persuade the participant that less pain from the test stimulus should be experienced during presentation of the second conditioning stimulus. After the 20 minute break, 10 test stimuli without conditioning were given to provide a second baseline measure (B2). The second conditioning stimulus was then applied and a further 10 test stimuli delivered (CS2). Following this, participants were asked to report what their expectations of the 
second conditioning stimulus had been after the explanation had been provided by the researcher. The same scale $(-100$ to +100$)$ was used as previously.

\section{Data analysis}

EMG data from the test stimuli delivered at B1, CS1, B2, and CS2 were rectified and averaged. The nociceptive flexion reflex was measured by calculating the EMG root mean square amplitude (RMS) between $85-150$ ms post-stimulus in the averaged response. NPR scores for the 10 test stimuli were averaged in B1, CS1, B2, and CS2. The RMS and NPR scores were analysed using a two-way repeated measures ANOVA with factors of conditioning (baseline, conditioned) and CS type (CS1, CS2). To identify any potential differences between the two types of conditioning stimuli, regardless of order of presentation, the data also were also analysed using a two-way repeated measures ANOVA with factors of conditioning (baseline, conditioned) and CS type (ischaemic arm test, cold pressor test). Expectation ratings and conditioning stimuli NPR scores were also compared between CS1 and CS2 using two-tailed paired T-tests. An alpha level of 0.05 was adopted for all analyses. Results are reported as mean \pm standard deviation.

\section{Results}

All participants completed the full session of testing without any adverse reactions to the test or conditioning procedures. Data from one participant were removed prior to analysis due to a severely abnormal increase $(140 \%)$ in nociceptive flexion reflex RMS during conditioning. In the remaining 19 participants, the average reflex threshold was $17.6 \pm 7.7$ $\mathrm{mA}$ and the average test stimulus intensity applied was $146 \pm 22 \%$ of reflex threshold. The two sets of baseline (test stimulus only) stimuli gave rise to NPRs of $54 \pm 23$ and $51 \pm 22$ and 
nociceptive flexion reflex RMS of $0.030 \pm 0.016$ and $0.025 \pm 0.014$. While the two baseline reflex sizes were not significantly different $(P=0.1)$, the NPR associated with the second baseline test was significantly lower than the first $(P=0.02)$.

For CS1, 15 of the 19 participants expected an inhibition of the test pain with conditioning, with an average expectation rating of $-27 \pm 22 \%$. The actual effects of conditioning on the test stimulus are shown in Figure 1. The conditioning stimulus gave rise to a $23 \pm 29 \%$ reduction in reflex RMS $(P=0.01)$ and a $12 \pm 9 \%$ reduction in NPR $(P<0.001)$. For CS2, 15 participants again expected an inhibition of the test pain and the average reported value (29 $\pm 21 \%)$ was not significantly different from the expectation rating of CS1 $(P=0.7)$. The ANOVA investigating the effects of CS1 and CS2 on baseline NPR data revealed main effects of conditioning $\left(\mathrm{F}_{1,18}=25 ; P<0.001\right)$ and conditioning type $\left(\mathrm{F}_{1,18}=14 ; P=0.001\right)$, as well as a significant interaction effect $\left(\mathrm{F}_{1,18}=10 ; P=0.006\right)$. Further investigation of the interaction effect indicated that the per cent change in NPR with conditioning was significantly greater at CS2 (26 $\pm 22 \%)$ compared to CS1 (12 $\pm 9 \% ; P=0.003)$. For the flexion reflex RMS data, there was a significant main effect of conditioning $\left(\mathrm{F}_{1,18}=11 ; P=0.004\right)$, indicating that flexion reflex RMS was smaller during conditioning compared to baseline across both conditioning stimuli. The interaction effect was not significant $\left(\mathrm{F}_{1,18}=0.4 ; P=0.5\right)$.

\section{Insert Figure 1 about here}

CS1 and CS2 results were subsequently combined and separated into ischaemic arm test and cold pressor test conditioning stimuli and the ANOVAs from above repeated. For the NPR data there was a significant main effect of conditioning $\left(\mathrm{F}_{1,18}=22 ; P<0.001\right)$ but the main effect of conditioning type $\left(\mathrm{F}_{1,18}=3 ; P=0.1\right)$ and the interaction effect $\left(\mathrm{F}_{1,18}=0.9 ; P=0.4\right)$ 
were not significant. The same results were found for the flexion reflex RMS data; the main effect of conditioning $\left(\mathrm{F}_{1,18}=11 ; P=0.004\right)$ was significant but the main effect of conditioning type $\left(\mathrm{F}_{1,18}=0.1 ; P=0.8\right)$ and the interaction effect $\left(\mathrm{F}_{1,18}=0 ; P=0.9\right)$ were not. Therefore, the two types of conditioning stimulus did not give rise to any differences in the extent conditioned pain modulation.

\section{Discussion}

We have shown for the first time that conditioned pain modulation can be enhanced within a person using a psychological intervention. Interestingly, modification of pain ratings in our study occurred without any change in reported expectancy and did not involve a change in spinal level nociceptive processing. We therefore propose that the intervention initiated an analgesic mechanism effective at a supraspinal level that is unrelated to both reported expectancy and the descending inhibition of spinal nociception. These findings are discussed in more detail below.

The participants, contrary to our hypothesis and despite being told that CS2 would incur increased analgesia, did not change their rated expectation of the effects of CS2 on test pain. Changing the delivery of the intervention to a more convincing and in-depth medical explanation or using a separate blinded assessor to obtain this outcome measure may have resulted in a greater effect on reported expectation of analgesia. Nevertheless, CS2 gave rise to a significantly greater inhibition of perceived test pain than CS1, indicating that the intervention had an effect on participants that was not equated to self-reported expectation. Pain is a multidimensional experience that involves the interpretation of nociceptive information under the constant influence of memories, emotions, and cognitive 
processes. ${ }^{17,18}$ There is significant scope for these processes to influence the pain experience through the myriad of connections between limbic, prefrontal, and other supraspinal areas known to be involved in nociceptive processing. Notably, the orbitofrontal cortex, which is strongly implicated in the supraspinal component of conditioned pain modulation, ${ }^{3,4}$ has reciprocal connections with many of these areas and provides a substrate for altered perceptions and emotions to modify the conditioned pain response. Several cortical regions are influenced by anxiety and expectancy regarding pain $^{19-22}$ and, more specifically, activity in the prefrontal cortex and anterior insula is modulated during manipulation of pain expectancy. ${ }^{23}$ Thus, cortico-cortical influences between these regions and the orbitofrontal cortex may mediate the interpretation of nociceptive information in response to perceptions of potential pain.

Given that our participants did not report a change in reported expectation, the effects of the intervention may be more likely to be mediated via a subconscious network. In a recent study, Jensen and colleagues ${ }^{24}$ showed that placebo and nocebo analgesia and hyperalgesia, respectively, could be induced using nonconscious cues. That is, participants did not consciously perceive the conditioning cues that gave rise to the analgesic and hyperalgesic responses. Given the nonconscious nature of conditioning, it was speculated that this response may involve subcortical brain regions, including the amygdala and striatum. Such structures also may be involved in the modulation of pain report in our study given that our participants did not report a conscious change in expectations of conditioning. Recently, Nir and colleagues ${ }^{8}$ showed that cognitive manipulation of the painfulness of the conditioning stimulus was able to reduce, but not increase, the conditioned pain modulation response in a healthy population. These authors hypothesised that a ceiling 
effect may have occurred in that pain inhibition was already saturated at pre-intervention. We did not see this ceiling effect in our study. This may reflect the modest level of pain inhibition achieved with CS1 in our study compared to the baseline response in Nir et al. ${ }^{8}$ Alternatively, it may indicate that expectations regarding the test pain, even subconscious ones, are more influential in modifying the conditioned pain modulation response than expectations regarding the conditioning pain.

We did not detect a change in spinal level processing of nociceptive information in our study despite the significant modulation of perceived pain. While several studies have shown a separation of segmental reflex and subjective pain during conditioned pain modulation, ${ }^{25-27}$ Goffaux and colleagues ${ }^{5}$ were the first to report a dissociation between the effects of expectation on spinal and supraspinal nociceptive processing in people with fibromyalgia. Expectation of analgesia with conditioning resulted in reduced perception of pain and diminished cortical potentials despite a facilitation of the nociceptive flexion reflex response at a spinal level. Similar to our argument above, the authors postulated that this dissociation was likely to reflect supraspinal processing of the ascending nociceptive response resulting in modulation of perceived pain. We did not record cortical potentials in our study so we are unable to comment on the ascending nociceptive signal beyond the level of the spinal cord. Nevertheless, although flexion reflex size and perceived pain ratings were significantly inhibited with conditioning both pre- and post-intervention, the magnitude of flexion reflex inhibition did not change following the intervention despite an increase in the magnitude of perceived pain inhibition. Therefore, we propose that our intervention did not influence activation of the descending DNIC system, inferring that modulation of the ascending nociceptive input was restricted to supraspinal levels. 
We used two different forms of conditioning stimuli in the current study so that the participant's experience of the first stimulus had a minimal influence on their expectations of the second. The effect of conditioning on flexion reflex RMS and the NPR were not significantly different between the two types of conditioning stimuli, indicating that an equivalent level of conditioned pain modulation was achieved. Furthermore, the order of the conditioning stimuli was randomised for each participant, making it highly unlikely that the type of conditioning stimulus influenced our main finding. A potential limitation of the study design is that the noted changes with CS2 may have been due to the fact that it was presented second, and not related to the intervention. While acknowledging this limitation, we think this is unlikely as the within session stability of the flexion reflex ${ }^{28}$ and conditioned pain modulation using both the ischaemic arm and cold pressor tests ${ }^{29}$ has been demonstrated previously. A further potential limitation of the study is that the test stimuli were not deemed painful by some participants, as the average NPR was just above 50 in both conditions. However, significant inhibition of the test NPR occurred with both conditioning stimuli. Thus, this factor is unlikely to have altered the outcome of the study.

In conclusion, informing participants that they were going to experience substantial analgesia from a novel conditioning stimulus resulted in a significantly larger decrease in perceived pain compared to the naive condition, despite no change in reported expectation. This is the first time that an increase in conditioned pain modulation has been reported following a psychological intervention targeting pain expectation. That additional analgesia can be achieved by such manipulation highlights the powerful influence of psychological factors on supraspinal pain processing and raises the potential that pain inhibition could 
also be enhanced in chronic pain populations who show impaired conditioned pain

modulation. ${ }^{30}$

\section{Acknowledgements}

Funding for this study was provided in part by an AUT University Student Assistantship Grant. 


\section{References}

1.Le Bars D. The whole body receptive field of dorsal horn multireceptive neurones. Brain Research Reviews. 2002;40(1-3):29-44.

2.Villanueva L, Bouhassira D, Le Bars D. The medullary subnucleus reticularis dorsalis (SRD) as a key link in both the transmission and modulation of pain signals. Pain. $1996 ; 67: 231-240$.

3.Moont R, Crispel Y, Lev R, Pud D, Yarnitsky D. Temporal changes in cortical activation during conditioned pain modulation (CPM), a LORETA study. Pain. 2011;152(7):14691477.

4.Piche M, Arsenault M, Rainville P. Cerebral and cerebrospinal processes underlying counterirritation analgesia. J Neurosci. November 11, 2009 2009;29(45):14236-14246.

5.Goffaux P, de Souza JB, Potvin S, Marchand S. Pain relief through expectation supersedes descending inhibitory deficits in fibromyalgia patients. Pain. 2009;145(1-2):18-23.

6.Goffaux P, Redmond WJ, Rainville P, Marchand S. Descending analgesia - When the spine echoes what the brain expects. Pain. 2007;130(1-2):137-143.

7.Larivière M, Goffaux P, Marchand S, Julien N. Changes in pain perception and descending inhibitory controls start at middle age in healthy adults. Clin J Pain. 2007;23(6):506510.

8.Nir R-R, Yarnitsky D, Honigman L, Granot M. Cognitive manipulation targeted at decreasing the conditioning pain perception reduces the efficacy of conditioned pain modulation. Pain. 2012;153(1):170-176.

9.van Wijk G, Veldhuijzen DS. Perspective on diffuse noxious inhibitory controls as a model of endogenous pain modulation in clinical pain syndromes. J Pain. 2010;11(5):408-419. 
10.Yarnitsky D. Conditioned pain modulation (the diffuse noxious inhibitory control-like effect): Its relevance for acute and chronic pain states. Curr Opin Anaesthesiol. 2010;23(5):611-615.

11.Edwards RR, Fillingim RB, Ness TJ. Age-related differences in endogenous pain modulation: A comparison of diffuse noxious inhibitory controls in healthy older and younger adults. Pain. 2003;101(1-2):155-165.

12.Tousignant-Laflamme Y, Marchand S. Excitatory and inhibitory pain mechanisms during the menstrual cycle in healthy women. Pain. 2009;146(1-2):47-55.

13.Sandrini G, Serrao M, Rossi P, Romaniello A, Cruccu G, Willer J. The lower limb flexion reflex in humans. Prog Neurobiol. 2005;77(6):353-395.

14.Rhudy JL, France CR. Defining the nociceptive flexion reflex (NFR) threshold in human participants: A comparison of different scoring criteria. Pain. 2007;128:244-253.

15.France CR, Rhudy JL, McGlone S. Using normalized EMG to define the nociceptive flexion reflex (NFR) threshold: Further evaluation of standardized NFR scoring criteria. Pain. 2009;145(1-2):211-218.

16.Charron J, Rainville P, Marchand S. Direct comparison of placebo effects on clinical and experimental pain. Clin J Pain. 2006;22(2):204-211.

17.Petrovic P, Ingvar M. Imaging cognitive modulation of pain processing. Pain. 2002;95:1-

5.

18.Tracey I, Mantyh PW. The cerebral signature for pain perception and its modulation. Neuron. 2007;55(3):377-391. 
19.Keltner JR, Furst A, Fan C, Redfern R, Inglis B, Fields HL. Isolating the modulatory effect of expectation on pain transmission: A functional magnetic resonance imaging study. $J$ Neurosci. 2006;26(16):4437-4443.

20.Franciotti R, Ciancetta L, Della Penna S, Belardinelli P, Pizzella V, Romani GL. Modulation of alpha oscillations in insular cortex reflects the threat of painful stimuli. Neuroimage. 2009;46(4):1082-1090.

21.Paulus MP, Stein MB. An Insular View of Anxiety. Biol Psychiatry. 2006;60(4):383-387.

22.Porro CA, Cettolo V, Francescato MP, Baraldi P. Functional activity mapping of the mesial hemispheric wall during anticipation of pain. Neuroimage. 2003;19(4):1738-1747.

23.Koyama T, McHaffie JG, Laurienti PJ, Coghill RC. The subjective experience of pain: Where expectations become reality. Proc Natl Acad Sci U S A. September 6, 2005 2005;102(36):12950-12955.

24.Jensen KB, Kaptchuk TJ, Kirsch I, et al. Nonconscious activation of placebo and nocebo pain responses. Proceedings of the National Academy of Sciences. September 25, 2012 2012;109(39):15959-15964.

25.Terkelsen AJ, Andersen OK, Hansen PO, Jensen TS. Effects of heterotopic- and segmental counter-stimulation on the nociceptive withdrawal reflex in humans. Acta Physiol Scand. 2001;172(3):211-217.

26.Bouhassira D, Danziger N, Atta N, Guirimand F. Comparison of the pain suppressive effects of clinical and experimental painful conditioning stimuli. Brain. May 1, 2003 2003;126(5):1068-1078.

27.Goffaux P, Michaud K, Gaudreau J, Chalaye P, Rainville P, Marchand S. Sex differences in perceived pain are affected by an anxious brain. Pain. 2011;152(9):2065-2073. 
28.Lewis GN, Rice DA, Jourdain K, McNair PJ. Influence of stimulation location and posture on the reliability and comfort of the nociceptive flexion reflex. Pain Res Manag. 2012;17(2):110-114.

29.Lewis GN, Heales L, Rice DA, Rome K, McNair PJ. The reliability of the conditioned pain modulation paradigm to assess endogenous inhibitory pain pathways. Pain Res Manag. 2012;17(2):98-102.

30.Lewis GN, Rice DA, McNair PJ. Conditioned pain modulation in populations with chronic pain: A systematic review and meta-analysis. J Pain. 2012;13(10):936-944. 


\section{Figure caption}

Fig. 1. Group results showing the effects of conditioning on the numerical pain rating (NPR;

top) and flexion reflex root mean square amplitude (RMS; bottom) for CS1 and CS2. A significant interaction in the pain rating data indicated a greater per cent reduction in NPR score with CS2 compared to CS1. CS = conditioning stimulus. Error bars represent one standard error of the mean. 
Paing Rating

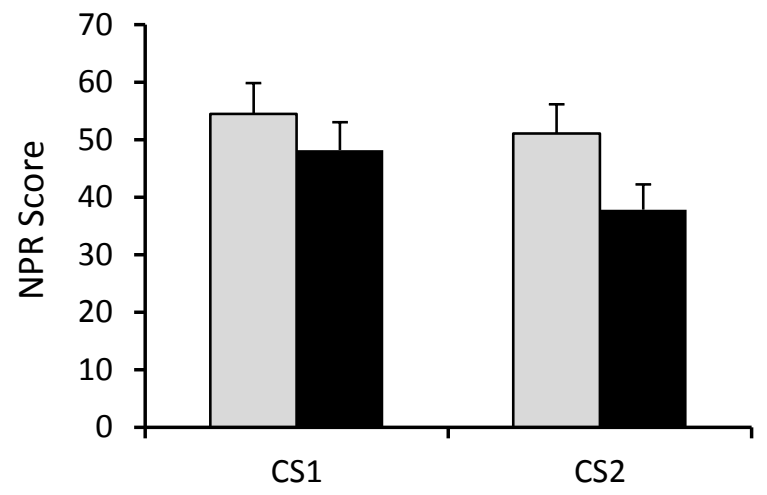

Flexion Reflex Size

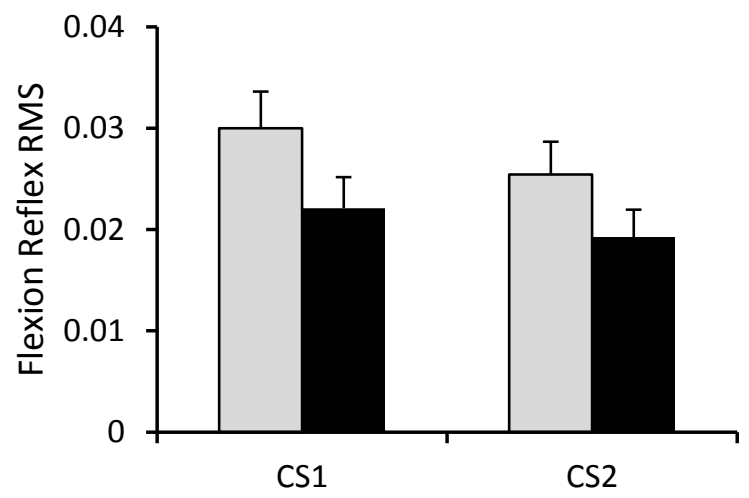

